\title{
Inducing Models of Human Control Skills
}

\author{
Rui Camacho ${ }^{1}$ \\ LIACC \\ FEUP \\ Rua do Campo Alegre, 823 Rua dos Bragas \\ 4150 PORTO \\ Portugal \\ 4099 PORTO CODEX \\ Portugal \\ Fax: (+351) $26003654 \quad$ Fax: $(+351) 2319280$ \\ e-mail: rcamacho@fe.up.pt \\ www : http://www.ncc.up.pt/ tau
}

key words: behavioural cloning, decision trees, cognitive modeling

\begin{abstract}
A new model of human control skills is proposed and empirically evaluated. It is called the incremental correction model and is more adequate for reverse engineering human control skills than any other previously proposed models. The experimental results show a considerable increase in robustness of the controllers that use the new model. The new model also attenuates the problem of unbalanced classes, noticed already in previous experiments. By means of Parameterised Decision Trees, propositional learners are still usable within the new model's framework.
\end{abstract}

\section{Introduction}

The problem addressed by the reported investigation concerns the reverse engineering of human control skills, also known as behavioural cloning. The problem specification attends the following restrictions. Machine Learning (ML) algorithms are to be used to induce the controllers. The controllers constructed by the ML algorithm, using performance data, should be induced automatically, intelligible to human understanding and should replicate the robustness features of the human subject being modeled.

An automatic process of reverse engineering human control skills offers a useful process of fast construction of controllers, specially in tasks where traditional Control Theory is not applicable. As pointed out by [10] it is also a very useful tool for training student pilots, particularly with regard to determining the aptitude of a candidate at an early stage of training. Hamm 92 ([7]) refers that Westland Helicopters Ltd uses helicopter engineering simulations, controlled by pilot models, for rotor loads and performance prediction studies. Because a human pilot is not included in the control loop, it is not necessary for the helicopter simulation to run in real time - performance models may be run faster than real time for chart data production, and complex models may be run at less than real time, in cheap workstations, for applications such as rotor load prediction studies. Urbančič et al([11]) describe the application of the reverse engineer methodology to the control of a crane in loading/unloading ships in a harbor. 
The problem of reverse engineering human control skills encounters several challenges. Since a real-time control strategy can not be articulated by the human subject, the model and its parameters must be conjectured and experimentally evaluated. The datasets that result from the behavioural traces have a set of features that make them "hard" for ML algorithms. The dataset is usually of large proportions, consisting of thousands of examples, and most of the attributes are real valued. The data is, usually, very noisy due to the fact that humans do not provide ideal control actions. There is also a natural unbalance in the dataset classes. Most of the time the system is near an equilibrium state and therefore, most of the time the controls do not change. Less often there are strong deviations from the desirable situation and the control values have to be changed substantially.

The controllers synthesised by Sammut et al.([10]) and [9][5][1] have the following limitations. They are not robust and the same controller is not adequate to fly different flight plans. The Decision Trees that constitute the controllers are very large (thousands of nodes). The model and ML tool parameters are hand tuned.

The reported work overcomes the mentioned limitations by proposing a new cognitive model for human control skills that include the use of goals. The new model has an underlying control strategy based on a sequence of adaptive control actions. Each control action, apart from the first of the sequence, corrects the previous action after its effects on the controlled system have been perceived. The experimental results show that the new model produces a significant improvement in the controllers robustness and a significant reduction in the complexity of the trees that code the controllers. The use of goals in the model allows the same controller to be used with different task plans. The use of a wrapper $([8])$ facilitates the tuning of the model and the ML tool parameters.

The new framework prompts the improvements in the methodology for reverse engineering human control skills described in the next section.

The structure of the rest of the paper is as follows. In Section 2 the methodology for reverse engineering human control skills is described. Section 3 surveys the models of Human Control Skills and describes the new model in more detail. In Section 4 the evaluation of the model's performance is presented. The experiments description and the results obtained are reported in Section 5 . The last section draws the conclusions.

\section{Methodology}

The methodology for reverse engineering human control skills, as described in [10], consists in the following steps. 
(1) Characterisation of the system being controlled as a set of state and control variables, representatives of the system status and decisions made by the human controller. (2) Definition of a task plan as a temporal sequence of stages. (3) Execution of the control task by the human controller according to the task plan. While performing the control task the system's state and control variables are recorded at regular intervals (the behavioural traces). (4) Pre-processing of the trace files to produce the ML tool datasets. (5) Induction of the decision trees using the ML tool. One tree for each control variable and for each stage. (6) Postprocessing procedure by assembling all parts into an artificial controller. Apart from the induced code for determining the value of each control variable, there is a hand-coded part that is responsible for switching the set of trees whenever there is a stage change in the task plan. The artificial controller replaces the human subject in the control cycle.

In the pre-processing procedure the data undergoes a series of filtering operations. The first filter splits the trace data into stages. A dataset is then created for each control variable and for each stage.

Each dataset is then subject to another filter that transforms samples into ML cases. In each sample, the value of the control variable is associated with the state variables and the other control variables of a sample recorded some time before. That time lag accounts for the human perceive-act delay. The control variable of the data set constitutes the class and the state, and the other control variables constitute the attributes.

When the synthesised controller is run as an auto-pilot the perceive-act delay used in the pre-processing procedure is introduced in the control cycle.

The proposed improvement in the methodology, [4], covers two aspects. First, the usage of goals. Second, the use of a wrapper to facilitate the controller's construction process by tuning the ML and model's parameters.

The two kinds of goals, Achievable goals and Homeostatic goals, used in the models are imported from the work on AI planning by [6]. Achievable goals have a well-defined set of start and final states in the state space; arriving at anyone of the final state marks the achievement and termination of such a goal. These goals are the most common type in AI systems. Homeostatic goals are achieved continuously. They do not terminate when the system is in one of the final states; when changes occur, and the state has changed, activity to re-achieve the final state is re-initiated.

A set of basic operations should be defined ${ }^{1}$ using the two kinds of goals defined above. During a basic operation the homeostatic and achievable goals remain constant. The task plan is then defined as a sequence of basic operations. In the flight domain, for example, the basic operations are a straight and level flight, a levelled left turn, a climb right turn etc. In a levelled turn, for example, the homeostatic goals are the bank angle, the altitude and the climb rate and the achievable goal is the final heading. A flight plan is a sequence of straight climb, levelled left turn, levelled right turn, etc.

\footnotetext{
${ }^{1}$ as already suggested in [10] but not possible within the models used so far.
} 


\section{Models of Human Control Skills}

The model of the artificial controller used in the experiments of [10], [9] and [11] has a two-level hierarchy of control: a high level module and a low-level one. The high-level module is hand coded and its only role, so far, is to sequence the stages of the task plan. Within the new model, the high-level module is also responsible for establishing the context for the low-level module. It switches the low-level modules according to the stage of the task plan. The context is further specified by defining the goal values for the new stage. The low-level module has been implemented as a set of decision trees, one for each aircraft control and for each stage. At each stage only the trees constructed for that stage are active. This module is the only one induced from the behavioural traces and will be referred as the "model" from now on. In this section two types of models are considered and compared: the "Goals" model; and the "Incremental Correction" model.

\subsection{The "Goals" Model}

As can be seen in Figure 1 the Goals model extends the earlier model used in [10] and [9]. In the "Goals" model each control value is not only determined by the state and other control variables of the system, but also by the set of goal values defined for each stage of the control task as given by the equation:

control value $(t+1)=f($ state variables, goals, other control variables $)$

This model uses the goal values to compute features that represent deviations from the goal values and also their temporal derivatives.

To use goals in a model within a ML propositional setting, a new computational model is required. As the new computational model is an extension of Decision Trees, it is called Parameterised Decision Trees.

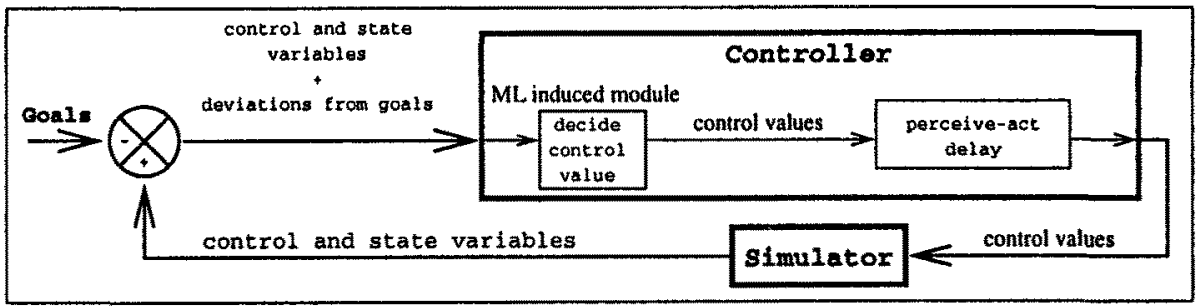

Fig. 1. "Goals Model".

A Decision Tree may be defined as a function from the space of attributes to the class space. A Parameterised Decision Tree is a function from the space of attributes and goals to the class space. The only difference between Decision 
Trees and Parameterised Decision Trees is in non-leaf nodes in which a continuous homeostatic attribute ${ }^{2}$ is used in a test. In such a node the difference between the attribute value and the corresponding goal value is computed before the comparison with the threshold is performed.

The Decision Tree construction algorithm is unchanged and therefore, any decision tree tool may be extended in this fashion. There is no loss in construction efficiency. What is required is a pre- and post-processing procedure. For details see [2].

A controller induced within the Goals model framework acts as a mapping from a situation (or range of situations) to a control device position (a value). This model, therefore requires that the control device position has to be memorise for each situation or range of situations. Which humans most certainly do not do.

\subsection{The Incremental Correction Model}

In order to understand the proposed control model consider the two situations illustrative of human real-time control skills. As a first illustrative situation consider a driver going in a car along a straight road and a second car going in front of him. Both cars drive at constant speed. Suddenly the car going ahead of him reduces considerably its speed. Perceiving the reduction in the distance between the two cars, the driver presses the break pedal. The amount of force applied is more or less, depending on the driver's initial judgment of the circumstances (distance reduction between the cars, speed differences, etc). After pressing the break pedal, the driver waits for the effect of the breaking to be perceivable and reevaluates the situation (distance between the cars, speed difference, etc). If the pressing was not sufficient the force is increased. If, on the other hand the speed reduction was excessive, the pressure is reduced. The driver repeats this procedure, of (re)evaluating the situation, adjusting the break pedal pressure and waiting for the effects of the previous action taken. The process stops when the situation is considered acceptable.

As a second illustrative situation consider an aircraft pilot flying straight and levelled when suddenly comes across an air sink. Perceiving the sudden loss in altitude the pilot undergoes a sequence of correction actions where each move of the control column corrects the previous move after its effects have been perceived. The sequence is stopped only when goal altitude is recovered.

Key aspects of the illustrative situations just described, and of general human real-time control, are as follows. There is a acceptable situation in which the controlled system is most of the time. The acceptable situation is characterised by a very small or non existing deviation from the homeostatic goal values. An acceptable situation requires no control change. Action is taken whenever the goal variables values deviate from the goal value. There is a reasonable "wild guess" to make the first correction, specially if the deviation is large. After the

\footnotetext{
${ }^{2}$ an attribute for which a homeostatic goal as been defined.
} 
first change, a sequence of (re)evaluation, corrections and waiting for the effects of the correction to become perceivable takes place until the acceptability of the situation is restored. The amount of waiting time involved in real-time control is usually very small. The control strategy is adaptive, in the sense that the direction and magnitude of the correction is directly affected by the previous change. If the previous change produces a too small reduction in the deviation then the direction of the next change is maintained and the amount of change increased. On the other hand, if the deviation is reduced too much or an overshooting is expected, then the direction is changed in the next cycle.

The equations to compute the control values within the IC model are:

$\operatorname{control}(t+1)=\left\{\begin{array}{cc}\text { control }(t), & \text { sit. ok } \\ \text { control }(t)+\Delta(\text { state vars, goals, other controls }), & \text { sit. } \text { not ok }\end{array}\right.$

As shown in Figure 2, the IC model has a module to determine if the situation requires a change in the controls, the Coarse Decision module. If a change in the controls is required (sit. not ok), then another module computes the values of the increment/decrement of the control variables, the Refined Decision module. Whenever a change in a control is made the controller uses a waiting time for the effects of control change to become perceivable. The IC model inherits the goals and the perceive-act delay from the Goals model.

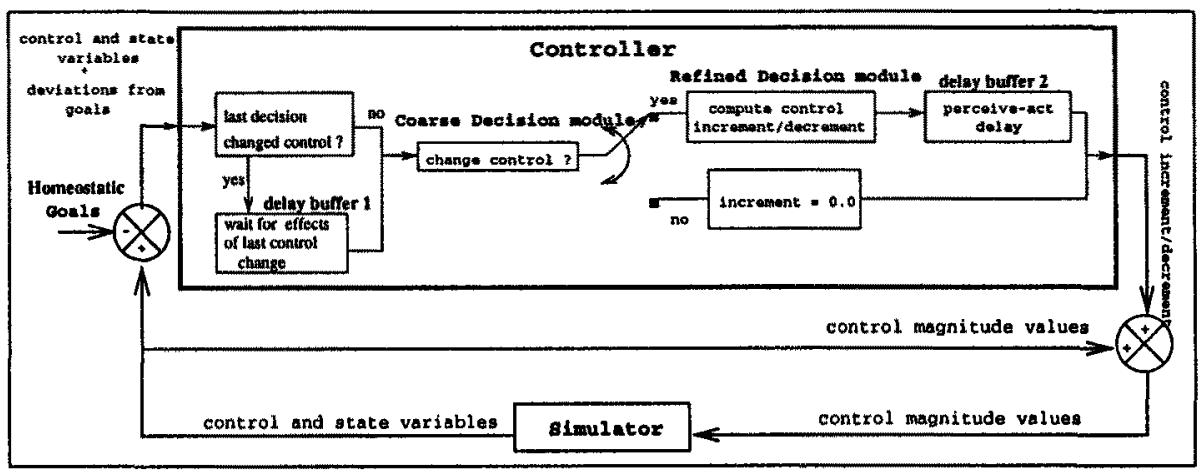

Fig. 2. Incremental Correction Model evaluated by the current experiments.

Both modules, Coarse Decision and Refined Decision, in Figure 2, are ML induced from the behavioural traces of the human subject as explained in Section 5. The Coarse Decision module implements the action/noaction decision making. The Refined Decision module determines the increment control value to apply whenever a control value has to be changed. The buffers 1 and 2, in the same figure, implement the "wait for effects" delay and the "perceive-act" delay respectively. 


\section{Performance Evaluation}

Reverse engineering human control skills may serve two aims. The first one concerns the construction of an accurate model of the human control skills on the given task. The second one concerns the automatic construction of a robust controller independently of being a good representative of the human behaviour. The first purpose will pay more attention to induction time measurements and to some tree statistics, whereas the second purpose will considers simulation time measurements as the crucial ones.

\section{Evaluation of Accuracy of the IC model}

The following error measures give an indication on how close the model is to the original system, the human controller.

The predictive power of the induced trees is estimated by measuring the Error Rate, the Mean Absolute Error (MAE), and the Root Mean Square Error (RMSE) on an independent test set. The Error Rate measures the percentage of errors made on the test set giving equal weight to each individual error. The MAE and RMSE are defined as

$$
M A E=\frac{\sum_{i=1}^{N}\left|c l s_{i}^{\prime}-c l s_{i}\right|}{N} \quad R M S E=\sqrt{\frac{\sum_{i=1}^{N}\left(c l s_{i}^{\prime}-c l s_{i}\right)^{2}}{N}}
$$

where $\operatorname{cls}_{i}$ is the actual class and $\mathrm{cls}^{\prime}{ }_{i}$ is the predicted value. Since the Coarse Decision module of the IC model outputs an action/no action decision, that is, a non numerical decision, only the Error Rate is used in this case.

The tree statistics that are gathered serve the purpose of estimating the interestingness of the tree as a cognitive model. One of the most desirable features of the constructed model is its intelligibility. To estimate such feature the tree size is measured.

\section{Flight simulation performance measurements}

The robustness of the controllers is estimated by the number of successful missions within the total used. A mission is successful if there is no crash between the initial and final points.

The flight smoothness is evaluated using a deviation measure associated with each of the three homeostatic attributes. For each homeostatic attribute the Mean Absolute Deviation (MAD) is measured using the definition

$$
M A D=\frac{\sum_{i=1}^{N}\left|a t_{i}-g \circ a\right| \mid}{N}
$$

Associated with each homeostatic goal there is a maximum acceptable deviation for that variable values. The frontier of the n-dimensional region containing the maximum acceptable values for the homeostatic variables is called the security envelope. All missions flown by the human subject that produce the behavioural traces were flown within the security envelope. The boundary for the altitude deviation is $100 \mathrm{ft}$, for the climb rate is $1000 \mathrm{ft} / \mathrm{min}$ and for the bank angle is $3^{\circ}$. The performance is evaluated by measuring the flight time percentage spent outside the envelope. 


\section{Experimental Settings}

The control task chosen for the experimental evaluation of the models consists in the control of a simulation of an F-16 aircraft performing a levelled left turn. A levelled turn is a nontrivial manoeuvre requiring a close coordination among the controls. The experiments were carried out using ACM 2.4 public domain flight simulator running on a HP Apollo Series 735 workstation. The author played the role of the human pilot necessary for this study. A detailed description of the empirical evaluation of the models can be found in [3] and [4].

The data used in the experiments are traces of 90 levelled left turn missions performed by the author. Aircraft state and control variables were sampled every 0.1 second. For the levelled left turn the achievable goal is the final heading. The homeostatic goals are the bank angle, the altitude and the climb rate. For each mission the achievable and homeostatic goals (except climb rate) were randomly generated from a range of admissible values. In all missions and at all times the aircraft was kept within the security envelope. The missions were flown by instruments only, the landscape was not rendered, reducing the possibility of the pilot to use features not measured (sampled) by the aircraft instruments. For a more detailed description of the experiments refer to [4] and [3]

The 90 missions are split into two halves, one for constructing the model and the other to estimate the predictive accuracy of the constructed model. The 90 missions contain approximately 481000 samples.

Following the methodology described in Section 2, the construction and test of the model is performed in three steps. In step one the pre-processing procedure converts the traces of the 90 missions into $\mathbf{C 4 . 5}$ data format. The data from the two sets of missions, training and testing, are always kept separate. The second step, using the wrapper, constructs the control trees by calling $\mathrm{C} 4.5$ and converts them into a Parameterised Decision Tree in $\mathrm{C}$ code. The control variables used in the experiments are the ailerons and the elevators control, which are enough to perform the levelled turn. One tree is constructed for each. The $\mathrm{C}$ code of both trees is joined to the rest of the controller code and linked to the simulator. In the third step, the model replaces the human pilot in the control loop of the simulator. The values for each control variables are obtained by interpreting the corresponding tree. The auto-pilot then "flies" all the 90 missions flight plans and the flight simulation performance measurements are made.

The wrapper algorithm, used in the second step, tunes the model's delays and the C4.5 $m$, cf and gain/gain ratio. The wrapper's search is described in detail in [4] and exploits the simplex method. The function to minimise is estimated by a 5 -fold cross validation procedure. In the construction of the Goals model the wrapper's guiding function is the RMSE. In the IC model the wrapper's function for the Coarse Decision module is the Error Rate and the RMSE for Refined Decision module. The perceive-act delay values used are $0.5 \mathrm{~s}, 1.0 \mathrm{~s}, 1.5 \mathrm{~s}$ and $2.0 \mathrm{~s}$. The values used for the "wait for effect" delay, in the IC model, are $0.0 \mathrm{~s}, 0.5$, and $1.0 \mathrm{~s}$. 
The attributes used in the construction of the controller's ailerons trees were: bank angle; bank angle derivative; bank angle acceleration; pitch; pitch rate; and the $\Delta$ elevators $(\mathrm{t})$. The attributes used in the construction of the controller's elevators trees were: altitude deviation; climb rate; climb rate derivative; bank angle; bank angle derivative; climb rate acceleration; and the $\Delta$ ailerons $(\mathbf{t})$.

For the IC model all samples are initially considered. The target definitions to learn within the IC model are: "change the control or not" and if the previous decision is favorable to change the control then the next decision is "what is the increment/decrement value to use". However the samples with no change in the control value largely outnumber the samples with "events" (the ratio is $36: 1$ ). The unbalance of such a data set is critical. The adopted procedure is as follows. In the first decision (action/no action) consider all the "event" as belonging to the same class (action) and all non-event samples as the other class (noaction). Build a data set with two classes equally represented by sampling from the noaction cases a number of cases equal to the action cases. The tree constructed with such dataset is then used to filter the "events" and produce the dataset for the Refined Decision module. In such a filtering procedure an event case is retained if it is predicted as action by the tree of the Coarse Decision module, otherwise is discarded. In the dataset of the Refined Decision module the increment of the control variable is restored as the class value.

\section{Results and Discussion}

\begin{tabular}{|c|c|c|c|c|c|c|c|c|}
\hline \multirow[t]{2}{*}{ Model } & \multicolumn{2}{|c|}{ crashes } & \multicolumn{2}{|c|}{ altitude (ft) } & \multicolumn{2}{|c|}{ climb rate $(\mathrm{ft} / \mathrm{min})$} & \multicolumn{2}{|c|}{ bank angle $\left(^{\circ}\right)$} \\
\hline & train set & test set & train set & test set & train set & test set & train set & test set \\
\hline Goals & 19 & 15 & 1806 & 1970 & 1664 & 1814 & $\overline{8}$ & $\overline{8}$ \\
\hline$\overline{\mathrm{IC}}$ & 0 & $\overline{0}$ & 36 & $\overline{34}$ & $\overline{95}$ & 94 & $\overline{0.5}$ & 0.5 \\
\hline Human & 0 & 0 & 21 & & & 94 & 0.7 & \\
\hline
\end{tabular}

Table 1. Flight simulation results. The results for altitude, climb rate and bank angle represent the average of the MADs. The average is computed using the successful missions and weighted with flight time.

The flight simulation performance of the two models together with the human subject performance are shown in Table 1. The entries for the altitude, climb rate and bank angle measurements in the table represent the average of the MADs. The average is computed using the successful missions and are weighted with the flying times. The flight simulation results for the Goals model controller show a considerable lack in robustness since only 26 missions were successful in the training set conditions and only 30 on the test set conditions. On the other hand, the IC model controller successfully flew the same 90 missions exhibiting therefore a better degree of robustness. The smoothness measure of the Goals 
model controller (MAD) also indicate that most of the time the aircraft is considerably far from the acceptable situation. For example, the average magnitude of the altitude is $1806 \mathrm{ft}$ which is eighteen times more than the altitude boundary of the security envelope that was never surpassed by the human controller. The smoothness results indicate that the IC model controller largely outperforms, the Goals model controller. The results of Table 1 also show that the IC model controller outperformed the human controller in two of the three measures (climb rate and bank angle).

\begin{tabular}{|c|c|c|c|c|c|}
\hline partition & $\mathrm{p} 1$ & $\mathrm{p} 2$ & $\mathrm{p} 3$ & $\mathrm{p} 4$ & $\mathrm{p} 5$ \\
\hline altitude (ft) & $35(14)$ & $73(68)$ & $85(57)$ & $27(10)$ & $39(7)$ \\
climb rate (ft/min) & $94(11)$ & $83(9)$ & $117(16)$ & $102(13)$ & $82(18)$ \\
bank angle ( ${ }^{\circ}$ ) & $0.5(0.1)$ & $0.6(0.1)$ & $0.5(0.1)$ & $1.4(1.4)$ & $0.5(0.1)$ \\
\hline
\end{tabular}

Table 2. Flight simulation MAD. $\mathrm{p}_{i}$ represent a different partition on the train/test set missions. The values are the mean and standard deviation over the 90 missions.

In order to gather more support for the claim that the IC model is better than the Goals model, four more controllers were constructed using different partitions of the 90 missions. The results of the all five IC model controllers are shown in Table 2. The entries in the table represent the mean and standard deviation over the 90 missions weighted by flying time. Each column in that table represents a different partition of the train/test set missions. All the controllers successfully flew the 90 missions and all of them largely outperform the Goals model controller. The results of all the five controllers are better than the original human controller in two of the three measures (climb rate and bank angle).

\begin{tabular}{|c|c|c|c|c|c|c|c|c|}
\hline \multirow[t]{2}{*}{ Model } & \multicolumn{4}{|c|}{ ailerons } & \multicolumn{4}{|c|}{ elevators } \\
\hline & t. size & Err Rate & RMSE & $\overline{\mathrm{MAE}}$ & t. size & Err Rate & RMSE & MAE \\
\hline Goals & 255 & 74.7 & $4.8 \mathrm{e}-4$ & & 3173 & 84.2 & $5.0 \mathrm{e}-3$ & \\
\hline \begin{tabular}{|l|l|}
$\mathrm{IC}$ & Coarse Dec. \\
& Refined Dec.
\end{tabular} & $\begin{array}{l}83 \\
663\end{array}$ & $\begin{array}{c}38.6 \\
-\end{array}$ & $4 . \bar{e}-4$ & - & $\begin{array}{c}65 \\
143\end{array}$ & $\begin{array}{c}42.4 \\
-\end{array}$ & 2.1e-3 & - \\
\hline
\end{tabular}

Table 3. Independent test set results. $t$. size stands for tree size. Coarse Dec. stands for Coarse Decision module. Refined Dec. stands for Refined Decision module.

The evaluation of the trees that constitute the controllers was done on an independent test set, and the results are shown in Table 3. With the exception of the ailerons of the Refined Decision module, the size of the trees of the IC model controller are much smaller than the corresponding tree size of the Goals model controller and of previous reverse engineering experiments [1]. The error rates of 
the IC model are nearly half the values found with the Goals model trees. The RMSE of the IC model trees are also smaller than the Goals model ones. The RMSE associated with the elevators trees is less than half the corresponding value of the Goals model.

We observe that the pruning facility of the ML tool may discard cases with class values that are used in exceptional situations (few examples in the data set), but are essential to recover from those exceptional situations. To measure how pruning parameters discard some classes less represented in the data and their relative importance the number of classes in the final tree is measured as well as the range of the classes that are used in the tree. The results are depicted in Table 4 and are taken from the elevators dataset of Goals model. The two levels of decision adopted in the IC model attenuates this pruning effect.

\begin{tabular}{|c||c|c|c|}
\hline $\mathbf{m}$ & range & n. classes & tree size \\
\hline 1 & {$[0.012: 0.078](100 \%)$} & $61(100 \%)$ & 10717 \\
5 & {$[0.012: 0.057](68 \%)$} & $42(69 \%)$ & 2563 \\
50 & {$[0.015: 0.051](55 \%)$} & $23(38 \%)$ & 199 \\
500 & {$[0.017: 0.027](15 \%)$} & $6(10 \%)$ & 21 \\
\hline
\end{tabular}

Table 4. Decrease in range and number of classes as C4.5's $\mathrm{m}$ parameter increases.

\section{Conclusions}

The use of goals and attributes that measure deviations from the defined goals is a significant improvement in the models. It makes possible to use data from different task plans when constructing the controllers and it allows the use of the same controller in different circumstances.

The main contribution of the IC model is a substantial increase in robustness of the new controllers. The flight simulation performance values are very close to the human subject performance on the same missions.

The trees constructed within the new model exhibit a smaller size than the ones from previous experiments in reverse engineering human control skills. Intelligibility of the models is an essential point in the success criteria and the small tree sizes are a good step towards their comprehensibility.

The new model avoids the "situation-control value" indexing mechanism underlying the previous models. The referred indexing mechanism would require a controller to have a memory of control positions for each situation(s), which is not realistic.

The main open problem found in the current investigation concerns the lack of correlation between the measures made on the independent test set and the flight simulation measures. If such a correlation can not be established there 
will be no guarantee that the set of the best trees, found by the wrapper, will produce a good controller. Automatisation of the controller construction process depends on such a correlation.

\section{Acknowledgments}

Thanks are due to Pavel Brazdil for valuable comments on this work. I thank also to Universidade do Porto and JNICT for the financial support during the PhD. Thanks are also due to Programa PRAXIS and FEDER and to the Programa de Financiamento Plurianual de Unidades de I\&D da JNICT.

\section{References}

1. R. Camacho. Laboratory note: On robustness tests of induced auto-pilots from single flight plan missions, Sept. 1994. Available from: http://www.ncc.up.pt/ tau.

2. R. Camacho. Laboratory note: Learning to turn: decision-trees to perform a levelled turn, Sept. 1995. Available from: http://www.ncc.up.pt/ tau.

3. R. Camacho. Laboratory note. an incremental correction model for reverse engineering human control skills, Oct. 1997. Available from: http://www.ncc.up.pt/ tau.

4. R. Camacho. Laboratory note. learning to turn: Parameterised decision trees to perform a levelled turn, June 1997. Available from: http://www.ncc.up.pt/ tau.

5. R. Camacho and D. Michie. Behavioural cloning: a correction. AI Magazine, 16(2):92, Summer 1995.

6. A. A. Covrigaru and R. K. Lindsay. Deterministic autonomous systems. AI Magazine, 12(3):110-117, fall 1991.

7. J. C. Hamm. The use of pilot models in dynamic performance and rotor load prediction studies. In Proceedings of the Eighteenth European Rotorcraft Forum, pages 15-18, Avignon, France, September 1992. Association Aeronautique et Astronautique de France.

8. H. G. John, R. Kohavi, and K. Pfleger. Irrelevant features and the subset selection problem. In Machine Learning: Proceedings of the Eleventh International Conference, pages 121 - 129, Rutgers Univ., New Jersey, July 1994. eds. William Cohen and Haym Hirsh.

9. D. Michie and R. Camacho. Building symbolic representations of intuitive realtime skills from performance data. In Machine Intelligence 13, pages 385-418. Oxford University Press, Oxford, United Kingdom, 1994. eds. K. Furukawa, D. Michie and S. Muggleton.

10. C. Sammut, S. Hurst, D. Kedzier, and D. Michie. Learning to fly. In Proceedings of the Ninth International Workshop of Machine Learning 92, pages 385-393, Aberdeen, U.K., July 1992.

11. T. Urbančič and I. Bratko. Reconstructing human skill with machine learning. In The Eleventh European Conference on Artificial Intelligence, pages 498 - 502, Amsterdam, Netherlands, 1994. 